\title{
A Report into the Requesting of Lyme Disease Tests in the Immunoserology Department at Nobles Hospital, Isle of Man
}

\section{Craig Pownall}

Medical Microbiology Laboratory, Noble's Hospital, Isle of Man.

*Corresponding author: Craig Pownall, Medical Microbiology Laboratory, Noble's Hospital, Strang, IM4 4RJ, Isle of Man, Email: craig.pownall@gov.im

Received date: June 24, 2018; Accepted date: August 12, 2018; Published date: August 21, 2018

Copyright: $@ 2018$ Pownall C. This is an open-access article distributed under the terms of the Creative Commons Attribution License, which permits unrestricted use, distribution, and reproduction in any medium, provided the original author and source are credited.

\begin{abstract}
Introduction: Lyme disease is caused by a spirochete infection of the genus Borrelia, transmitted through bites of the sheep tick Ixodes Ricinus on the Isle of Man. This can lead to a number of short-term non-specific symptoms, and longer-term debilitating effects.
\end{abstract}

Objective: This study analyzed the requesting of Lyme disease serology at the Immunoserology laboratory at Noble's Hospital in order to look for money saving opportunities.

Materials and Methods: A data grab was performed on the LIMS system in use at Noble's Hospital, Isle of Man, for Lyme disease testing requested over a five-year period, with results transferred to an Excel spreadsheet. This data was then sorted into various categories, including year-by-year and month-by-month totals, percentage of requests honored, source of requests, in-depth investigations of GP requests, and results of tests.

Results: Findings indicated that Lyme disease requesting had doubled over this five-year period, with over twothirds of requests being from General Practitioners. Several surgeries were found to be requesting significantly more than the proportion of the population served indicated was appropriate.

Discussion: In order to ensure that clinicians made suitable judgments, and therefore the best use of resources, this report makes three recommendations:

1. Communication sent to GP practices reminding them of the importance of testing only in the event of clinical suspicion, characteristic rash, and evidence of a tick bite. Limitations of testing and details of specialist advice from Rare and Imported Pathogens Laboratory (RIPL) also included.

2. RIPL contact information also added to the comment automatically applied to a negative sample. Comment added to positive samples regarding the limitations of the test, and RIPL contact details.

3. Information disseminated to the public regarding the Public Health England (PHE) Tick Surveillance Scheme in order to maintain epidemiological data.

\section{Introduction}

Three species of the Borrelia genus are known to be responsible for causing Lyme disease in humans; $B$. burgdorferi in the USA and Europe, and B. garinii and B. afzelii in Europe [1-3]. Early symptoms are mediated by an acute infective process with later manifestations related to the host immune response [3]. After inoculation, the organism migrates from the site via the blood or lymphatics, producing the characteristic initial expanding red macule or papule (erythema migrans) [2,3], although up to one third of cases may not have the clinically indicative rash [4]. Additional common early symptoms are flu-like, with a combination of aching, fever, headache, fatigue, sweating, joint pain, light and sound sensitivity, abnormal sensations, and stiff neck possibly being encountered [4]. Later symptoms may include headaches, conjunctivitis, fever, and regional lymphadenopathy $[2,3]$, with complications including skin lesions, myocarditis, arthritis, aseptic meningitis, and cranial nerve palsies possible [3]. The clinical case definition of Lyme disease is the presence of erythema migrans of approximately $5 \mathrm{~cm}$ diameter, or at least one late manifestation symptom and laboratory confirmation. However, clinical diagnosis is complicated by varied manifestations of the disease, as shown above, and a lack of reliable diagnostic tests [1]. Laboratory diagnosis is difficult due to the organism being microaerophilic with complex nutritional needs which, combined with a generation time of around 18 hours, makes it difficult to grow in the laboratory [1]. Additionally, Nucleic acid-based tests have a low sensitivity; $65 \%-75 \%$ with skin biopsies, 50\%-85\% with synovial fluid, and $25 \%$ with CSF have been reported [1]. Based on these limitations, a 2-stage approach to diagnosis is recommended; sero-diagnosis followed by Western blot confirmation [2,3]. Whilst serological testing is the initial test of choice for Lyme disease diagnosis, this is not without drawbacks. All serological tests are relatively insensitive during the early acute phase, with IgM taking [2-4] weeks to reach detectable levels after the onset of erythema migrans and peaking after 6-8 weeks, and IgG peaking after 4-6 months [1]. 
Page 2 of 6

Hard ticks of the genus Ixodes are the major vector for the causative organism of Lyme disease [3] with I. ricinus prevalent in Europe [1]. These ticks are a blood feeding ectoparasite of mammals, birds and reptiles, and can transmit a wide variety of pathogenic organisms [5]. As shown in Figure 1, the officially reported distribution of ticks in the British Isles, with the Isle of Man data based on 30 historical records with a resolution of $10 \mathrm{~km} \mathrm{[5].}$

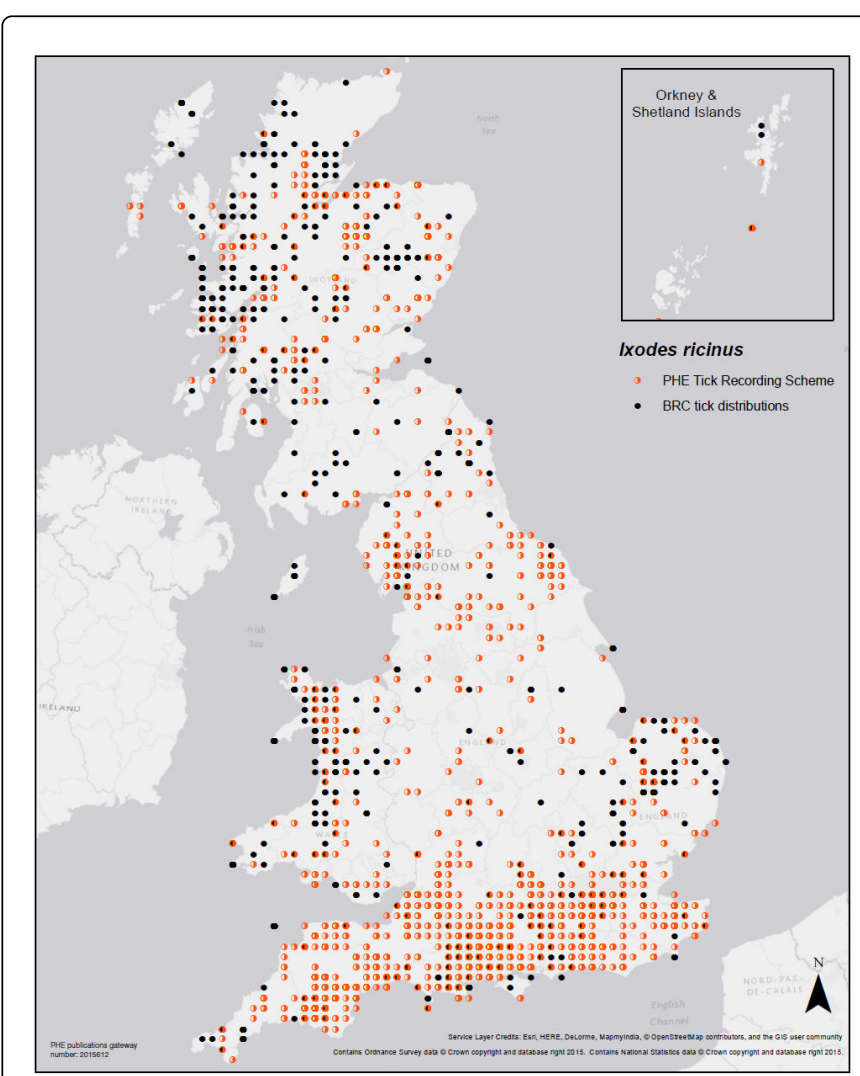

Figure 1: The distribution of $I$. ricinus in the British Isles, as reported by various tick reporting schemes [6].

Although Figure 1 shows only a limited amount of information regarding the Isle of Man other than historical presence, the Island does anecdotally harbor a large amount of $I$. ricinus ticks, especially in the Curraghs area [7].

In the British Isles, confirmed cases have increased in recent years. Mean annual incidence rates for laboratory-confirmed cases has risen from 0.38 cases per 100,000 in the years $1997-2000$ to 1.73 cases per 100,000 total population in 2011 [8]. Regarding current infection, confirmed cases between January and September 2017 are 1171, up from 867 in same period in 2016 [9], although there are estimated to be between 1,000 and 2,000 additional cases in England and Wales each year than those reported by laboratories [8]. Medical Microbiology laboratories have been required to notify diagnosis to Public Health England (formerly Health Protection Agency) since October 2010, with enhanced surveillance in the form of questionnaires sent to clinicians requesting additional data about laboratory-confirmed cases [8], which may account for some increase. Further factors attributed to an increase in cases of Lyme disease are increased awareness, greater access to diagnostic facilities and demand for such testing, more sensitive diagnostic methods, changes in human population sizes, changes in geographical ranges of ticks and weather patterns affecting tick numbers, and an increase in the popularity of activity holidays [8,9]. Additionally, an increase in migrants from Central and Eastern Europe since 2000 has seen a suspected increase of incidence of infection identified but not acquired in the UK, with an average of $15 \%$ of cases acquired abroad since this year [8].

\section{Objective}

An in-depth analysis with regards to the diagnostic requesting habits of the medical practitioners on Isle of Man and associated test results is to be used to elucidate recommendations in order to reduce the number of unnecessary requests and save money and resources.

\section{Materials and Methods}

The Isle of Man has a total area of 221 square miles and a population of approximately 85,000 . This population is served by a 314-bed hospital and 12 general practitioners' surgeries. The Immunoserology laboratory located in Noble's Hospital is staffed by one full-time and one part-time Biomedical Scientist, with a further three Biomedical Scientists being assigned into the department from the Medical Microbiology laboratory on a month-by-month rotation. This department is responsible for the testing of blood samples for virology and other infectious organism serology, and autoimmunity antibodies. Additionally, a full microbiological screening service is provided for the Isle of Man blood donor service. A full analysis was performed on the Lyme disease requesting data from this department from $1^{\text {st }}$ January 2013 to $31^{\text {st }}$ December 2017. A data grab was performed on the LIMS system in use (Masterlab, CliniSys Group Ltd.) for this fiveyear period, with results transferred to an Excel (Microsoft) spreadsheet. This data was then sorted according to various categories and converted to graph form using this same software package.

\section{Results}

As shown in Figure 2, year-by-year total of requests to the Immunoserology department at Noble's Hospital on the Isle of Man, clearly showing an increasing trend of requests being made.

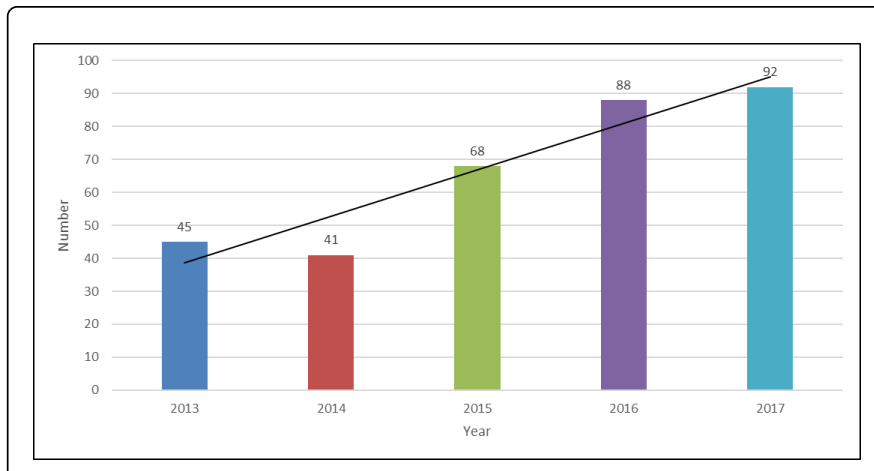

Figure 2: Number of Lyme disease testing requests made to the Immunoserology laboratory at Noble's Hospital on the Isle of Man for the years 2013-2017.

Over double the number of requests were made in 2017 as the years 2013 and 2014. Although corporate confidentiality precludes exact costing from being divulged, this resulted in a potentially expensive escalation of Lyme disease testing. However, not all requests were 
tested, with rejections of testing based on factors such as absence of clinical details or a lack of evidence to suggest that testing for this disease is appropriate, with the Consultant Microbiologist being directly involved in this decision-making process. As shown in Figure 3 , the numbers of requests being honored versus of those is rejected.



Figure 3: Percentage of Lyme disease test requests performed in the Immunoserology laboratory at Noble's Hospital on the Isle of Man for the years 2013-2017.

In total, $12 \%$ of test requests were rejected, giving a total of 294 tests being performed in the five-year period under scrutiny.

As to the timings of the requests through the year, approximately half of the cases of Lyme disease are diagnosed in specimens collected in July, August and September due to infection being acquired in the peak tick feeding season of late spring/early summer [10]. Figure 4 shows that, when averaged out over the 5-year period, the requesting of Lyme disease does indeed reach a peak in August.

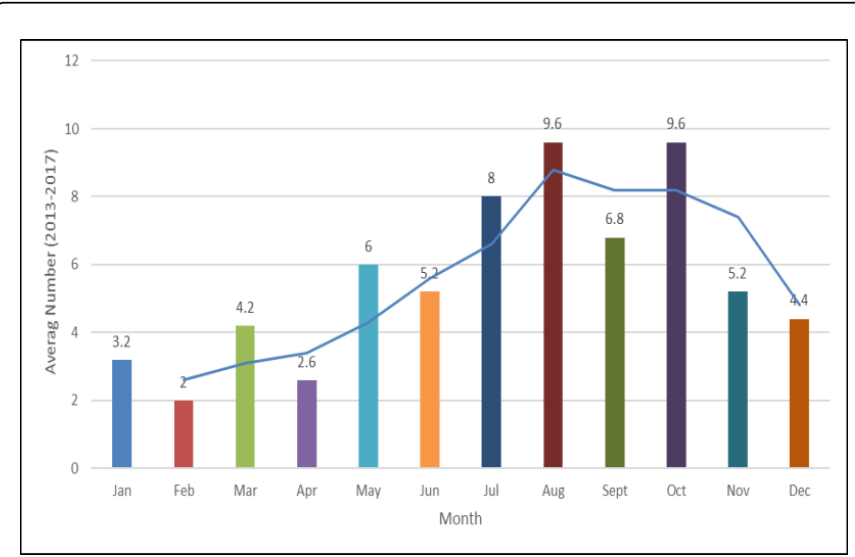

Figure 4: The number of Lyme disease test requests made to the Immunoserology laboratory at Noble's Hospital, Isle of Man by month, averaged over the 5 -year period.

This suggests that a good proportion of the requests may take into account the epidemiological time-frames of acquiring and incubating the disease. However, as shown in Figure 4, this is only an average. As shown in Figure 5, when taken by each individual per year, there are inconsistencies in the pattern.

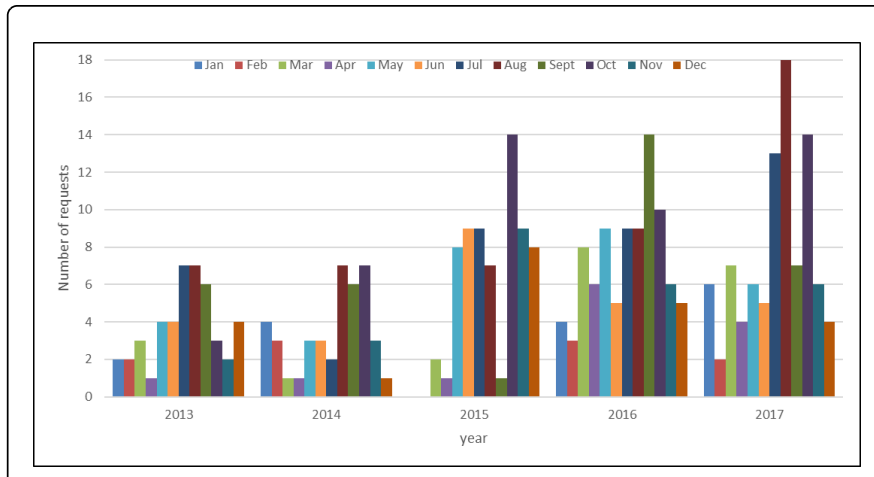

Figure 5: The number of Lyme disease test requests made to the Immunoserology laboratory at Noble's Hospital, Isle of Man by month, shown by year over the 5 -year period.

The $I$. ricinus tick is known to be historically dormant in the winter months [11]. However, 8 requests were made for Lyme disease testing in March 2016, whilst only 1 request was made in September 2015. This is the opposite of what would be expected at these times of year. Indeed, the September figures range from 1 request in 2015 to 14 requests in 2016. Opposing this is the fact that early symptoms are relatively non-specific, and therefore may not be acted upon by the patient if the characteristic skin abnormality is not present. The patient may only present months or even years after becoming infected when secondary symptoms become apparent, thereby rendering the timing of seeking medical assistance is inconsequential. It can be seen that both sets of reasoning mean that the month-by-month timings of requests in this study are meaningless and therefore will not figure in the conclusions made.

With regards to the source of the testing requests, over two-thirds (68.9\%) of requests were made by General Practitioners as shown in Figure 6.

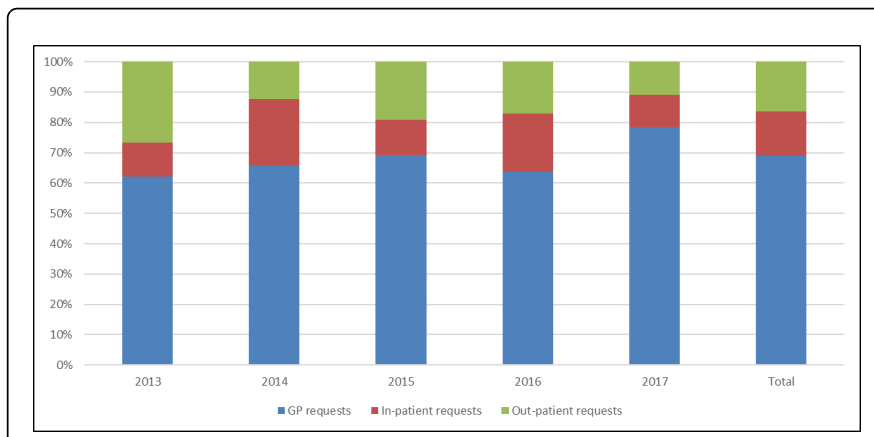

Figure 6: Lyme disease test requesting to the Immunoserology laboratory at Noble's Hospital, Isle of Man sorted by year and source.

As shown in Figure 7, bearing this in mind, it was deemed prudent to compare the percentages of requests made by each GP surgery with the population of the Isle of Man that they serve in order to ascertain if there are any surgeries that are sending significantly more requests than the proportion of the population that they suggest serving. 


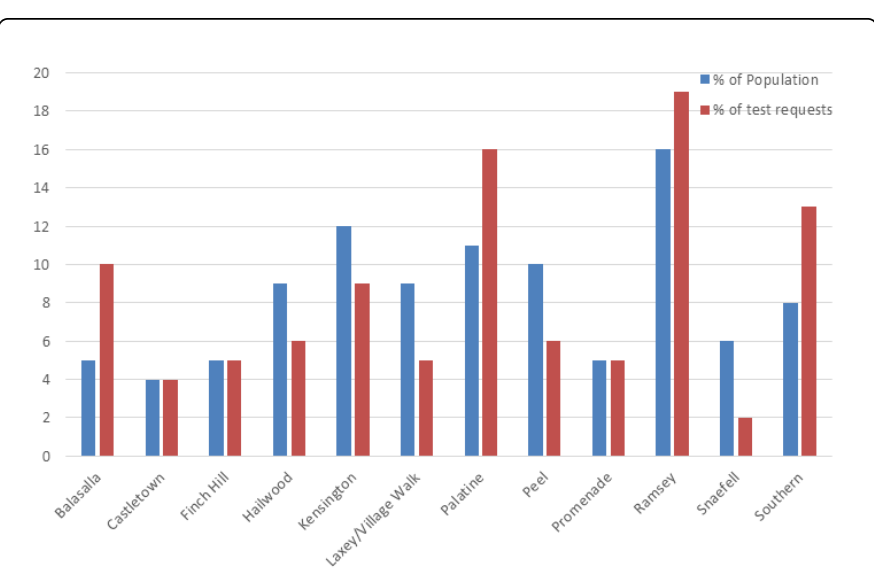

Figure 7: Percentage of the population of the Isle of Man served by each GP surgery (correct as of December 2017) versus the percentage of Lyme disease testing requests by each surgery for the time period 2013-2017.

The information presented above shows that four out of the twelve GP surgeries on the Isle of Man have sent more Lyme disease requests over the period 2013-2017 than the proportion of the population registered there suggest that they should; Ballasalla $(+100 \%)$, Southern $(+63 \%)$, Palatine $(+45 \%)$ and Ramsey $(+19 \%)$. However, before making a judgment about this, it is necessary to preclude any confounding factors that may contribute to the increase in requesting.

Geographical distribution of surgeries may be excluded as a confounding factor due to the small size of the Island and lack of appreciably different climate ranges therein. Another factor that may influence these figures is the age of the patients served by each GP practice; the age range most likely to be infected with Lyme disease being 45-64 [10]. Table 1 shows a comparison of patients within the primary age range for Lyme disease in the four above GP surgery populations as well as two surgeries with a lower than expected request rate.

\begin{tabular}{|l|l|l|}
\hline GP Surgery & $\begin{array}{l}\text { \% of patients in the } \\
\mathbf{4 5 - 6 4} \text { age range }\end{array}$ & $\begin{array}{l}\text { Increase/decrease in } \% \\
\text { of test requests based } \\
\text { on \% of population } \\
\text { served }\end{array}$ \\
\hline Ballasalla & $32.8 \%$ & $+100 \%$ \\
\hline Southern & $29.5 \%$ & $+63 \%$ \\
\hline Palatine** & $28.1 \%$ & $+45 \%$ \\
\hline Ramsey & $29.4 \%$ & $+19 \%$ \\
\hline Peel & $28.6 \%$ & $-40 \%$ \\
\hline Laxey/Village Walk & $30.3 \%$ & $-44 \%$ \\
\hline
\end{tabular}

Table 1: A comparison of the primary age range for Lyme disease in GP surgery populations with the increase/decrease in the percentage of test requests based on the percentage of population served. ${ }^{\star}$ Based on Isle of Man 2016 Census [12]. ${ }^{*}$ Palatine information based on whole of Douglas results, due to no information being available for separate areas of Douglas
As can be seen from Table 1, the areas of the Isle of Man looked at here have a relatively homogenous population within the 45-64-yearold age range, with no GP surgery having a significant variation away from the mean of $29.8 \%$. This can be taken as evidence that the age of the populations served by each GP surgery is not a factor in the differences in the expected percentage of test requests based on the percentage of the Island population served. Thus, Ballasalla, Southern, Palatine and Ramsay General Practices can be seen as making a higher number of Lyme disease requests than may be necessary.

Figures 8 and 9 shows the results of Lyme disease testing in the Immunoserology department at Noble's Hospital, Isle of Man during the time period 2013-2017, expressed as numbers and proportions respectively.



Figure 8: Lyme disease test results from the Immunoserology laboratory at Noble's Hospital on the Isle of Man for the years 2013-2017.

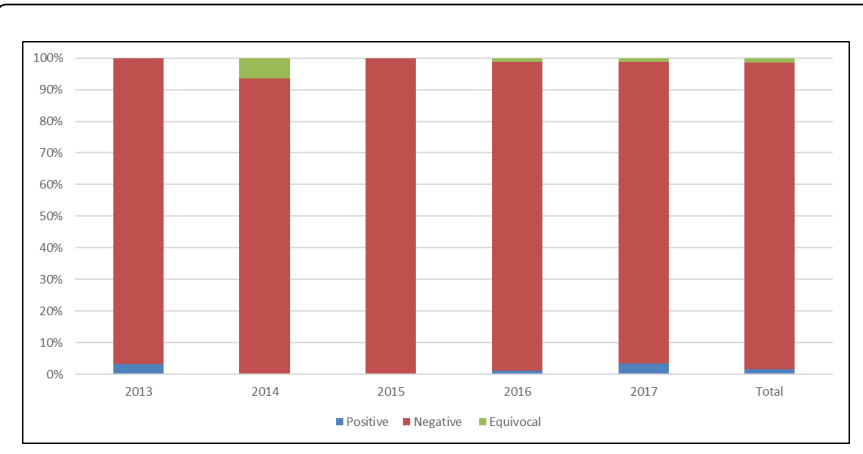

Figure 9: Lyme disease test results from the Immunoserology laboratory at Noble's Hospital on the Isle of Man for the years 2013-2017.

\section{Discussion}

As can be seen, the number of negative results far outweighs the number of positive and equivocal results on a consistent basis. Indeed, 2015 showed a $100 \%$ negative rate. With regards to three equivocal results obtained during the whole 5-year period under scrutiny, two were sent away to the Rare and Imported Pathogens Laboratory (RIPL) at Porton Down who reported that these may be non-specific reactions, but that recent infection could not be ruled out. However, both patients tested negative on repeat sampling. The third sample was also sent away for confirmation, with the RIPL stating that there was 
no evidence of Lyme disease, and to consider the sample negative. The sample from a fourth equivocal result was not sent away due to being a short sample, with a repeat requested which did not materialize. This sample therefore cannot be submitted as evidence of being a positive or negative result. However, the fact that the clinician did not deem it relevant to send a repeat sample suggests that a differential diagnosis had been performed using other test parameters, with Lyme disease no longer under suspicion. During the five-year period of the study, 5 samples were found to be positive. These samples were also sent away to the RIPL for serological confirmation and Immuno-blot analysis. The results from the two positive samples from 2013 and 2016 were reported as "early infection" and "consistent with very recent infection" respectively. These patients were treated accordingly. These results were obtained from specimens taken in September (2013) and July (2016), consistent with the April-October tick feeding season. Whilst there is no information available regarding where the patient that tested positive in 2013 may have been infected, the patient that tested positive in July 2016 was found to have been bitten by a tick in Sweden. This confirms that this incident was not as a result of infection on the Isle of Man. As an aside, it is interesting to note that this patient suspected Lyme disease after being bitten by a tick in early June 2016, but found her suspicions dismissed by a doctor in Noble's Hospital A\&E department despite the characteristic erythema migrans rash and other appropriate signs and symptoms. The first blood test from this patient was negative for Lyme disease, with a second sample ordered by a more sympathetic GP after four weeks being positive, thus demonstrating the potential disadvantages of Lyme serology, especially in the early stages of the disease. The results from the three positive samples from 2017 were all confirmed by the RIPL to be consistent with past Lyme disease. Although three samples were found to be positive from 2017, this represents only two patients, with one positive sample being a repeat sample. The patient found positive repeatedly in July and August 2017 was found to have had no off-Island travel, and had spent time walking in the Manx Glens. It therefore looks likely that this patient contracted the infection on the Isle of Man.

Due to the intrinsic problems of serological testing for Lyme disease, as demonstrated above, when prevalence of Lyme disease is low, for example on the Isle of Man, the likelihood of a positive test result being a true positive is low (PPV $<10 \%)$. It is therefore recommended that serological testing should only be performed with highly suggestive clinical findings [2], with the absence of an appropriate history and clinical suspicion of Lyme disease precluding the testing [1]. Whilst this is done on a limited basis at the moment by the consultant microbiologist once specimens arrive at the laboratory, the onus should be placed on the clinicians to make more informed choices prior to a sample even being taken.

It may therefore be desirable to send out communication to at least the GP practices reminding them of the importance of testing only in the event of clinical suspicion, characteristic rash, and evidence of a tick bite. This communication may be based upon a recently released letter to clinicians from the Rare and Imported Pathogens Laboratory (RIPL) regarding Lyme disease testing [13], with limitations of the test included on this communiqué, in order to further inform the clinicians of the reasons for prudence in this area. Details for specialist medical advice from the RIPL should also be included in order for clinicians to seek advice prior to considering Lyme disease as a serious possibility [4].

The second recommendation relates to laboratory comments attached to reports when testing has been requested and completed. It is recommended that no further testing is required in the case of a negative result [1]. However, if $\operatorname{IgM}$ and $\operatorname{IgG}$ tests are negative, a comment is currently added stating, "This does not rule out recent infection. Treat erythema migrans on clinical suspicion. If recent infection is suspected, consider sending follow-up serology 4-6 weeks after original sample, although prompt antibiotic treatment may abrogate the antibody response. If chronic infection is suspected no further action is needed" [14].

This is based upon Public Health England recommendations which states that if the initial test is negative, but symptoms persist, a repeat sample should be sent 3 to 4 weeks after the initial test [4]. However, it could be advantageous here to include RIPL contact information and recommend this be the next step prior to repeating the sample. The specialist medical advice given by the RIPL may dissuade the clinician from sending follow up samples, thus saving money and resources [4].

If IgM and/or IgG are positive, the sample is sent to the RIPL for confirmation and a report is sent out as screen positive with a laboratory comment attached to the report stating as such, with a further report to follow [14]. However, perhaps information regarding the disadvantages of testing, for example the risk of false positive results, could be included at this point. Again, RIPL contact information may be included here in order to take advantage of the specialist advice therein.

The third recommendation regards the Tick Surveillance Scheme. This Public Health England-run scheme records tick distributions on a national scale, and how populations may be changing, via the sending of collected ticks along with a raft of supplementary information such as details of date of collection, location grid reference, nearest town/ village, and the host that the tick was attached to [6]. The Tick Surveillance Scheme details could be disseminated via a newspaper article, or through leaflets located in GP surgeries or woodland public buildings in order to inform the general public, and ensure epidemiological data is maintained.

However, one potential drawback of this is that pathogens are not tested for in collected ticks due to seasonal/geographic variations in rates of transmission, and the presence of the pathogen not necessarily meaning that the pathogen is passed on [6]. This data would therefore only be useful as one factor in informing the choices of clinicians as to the suitability of sending samples for Lyme disease testing.

\section{Conclusion}

Lyme disease is a potentially debilitating vector-borne infection from a species of the Borrelia genus of spirochetes passed on from the Ixodes tick. The characteristic symptom is the erythema migrans skin rash, combined with a variety of non-specific symptoms. Later manifestations of the disease can include arthritis, conjunctivitis and skin lesions.

The disease is diagnosed serologically, although this is not without disadvantages, with confirmation testing by Western Blot against the organism specific antigens. Although there is historical evidence of the vector being present on the Isle of Man and a high probability of at least one case in this study being contracted here, there is insufficient evidence from this study as to the carriage of the spirochete, or to the transmission rate on the Island.

During the five-year period of 2013-2017, 288 samples tested negative (including the 3 equivocal samples that were later confirmed as negative), with 4 patients being confirmed as positive (the fifth, 
Citation: Pownall C (2018) A Report into the Requesting of Lyme Disease Tests in the Immunoserology Department at Nobles Hospital, Isle of Man. J Bacteriol Parasitol 9: 342. doi:10.4172/2155-9597.1000342

Page 6 of 6

duplicate positive sample being disregarded from the final figures) Thus, of the 292 samples included here, a highly conservative estimate of six times the cost of positive samples was spent on determining negative samples (including reference laboratory costs where applicable, but excluding labor/instrumentation costs, as well as the use of tests for instrument calibration as opposed to patient testing).

Whilst some testing may be justified in order to rule out the infection due to symptoms resembling the disease and a genuine suspicion of the infection, many cases may be seen as frivolous, with a "just in case" testing approach demonstrated. Thus, three recommendations corroborated by a number of evidence-based sources may be put forward here to cut down on these ill-considered testing requests in order to save the department, and thus the Isle of Man Government, money in the long-term.

\section{Declaration}

The author declares that no financial or personal interest has influenced the findings of this research.

\section{Acknowledgements}

The author wishes to thank all of the staff in the Medical Microbiology and Immunoserology departments at Noble's Hospital for all of their help and support during this research. Extra thanks must be given to the laboratory manager Rebbeca Shields for proofreading the reports, senior Biomedical Scientist Chris Helm for providing IT assistance and Consultant Microbiologist Dr. Rizwan Khan for initiating the study and providing assistance in compiling the information therein. Additional thanks to the Isle of Man Chief Veterinary Officer Alistair Breed for expert knowledge and advice provision.

\section{References}

1. Murray P, Rosenthal K, Pfaller M (2016) Medical Microbiology (8thedtn), Elsevier, Philadelphia, pp. 107-115.

2. Brooks GF, Carroll KC, Butel JS, Morse SA (2013) Jawetz, Melnick \& Adelberg's Medical Microbiology (26th edtn), McGraw-Hill Companies Inc., USA, pp. 149-246.

3. Gillespie S, Bamford K (2012) Medical Microbiology and Infection at a Glance (4thedtn), John Wiley \& Sons, Inc., USA, p. 147.

4. Public Health England (2015) Suggested Referral Pathway for Patients with Symptoms Related to Lyme Disease. PHE Publications e2014099.

5. Pietzsch ME, Medlock JM, Jones L, Avenell D, Abbott J, et al. (2005) Distribution of Ixodes ricinus in the British Isles: investigation of historical records. Med Vet Entomol 19: 306-314.

6. Public Health England (2016) Tick Surveillance Scheme. PHE Publications e2015700.

7. Pownall C (2018) Communications with Alistair Breed, Isle of Man Chief Veterinary Officer (01/02/2018-23/04/2018). Personal communications.

8. Public Health England (2013) Lyme Borreliosis Epidemiology and Surveillance. PHE Publications e20138562.

9. Public Health England (2017) Common animal associated infections quarterly report (England and Wales): third quarter, Health Protection Report. PHE Publications 11: 41.

10. Public Health England (2013) Lyme Disease Epidemiology and Surveillance. PHE Publications e2013683.

11. Dautel H, Dippel C, Kämmer D, Kahl O (2008) Winter activity of Ixodes ricinus in a Berlin forest. Int J Med Microbiol 298: 50-54.

12. Isle of Man Government (2016) Isle of Man Census Report. Isle of Man Department of Economic Affairs 17: 004.

13. Brooks T (2018) Notes for clinicians on laboratory tests for Lyme disease [letter]. Public Health England.

14. Hewson S (2016) IM-SOP-17 (v1.0) Lyme Disease IgG/IgM Testing on VIDAS 3 [Internal SOP]. Isle of Man Government 16: 006. 